\title{
POSITIVE LINEAR FUNCTIONALS WITHOUT REPRESENTING MEASURES
}

\author{
ChirakKal EASWARAN AND LaWrence Fialkow
}

Abstract. For $k$ even, let $\mathscr{P}_{k}$ denote the vector space of polynomials in 2 real variables of degree at most $k$. A linear functional $L: \mathscr{P}_{k} \longrightarrow \mathbb{R}$ is positive if $p \in \mathscr{P}_{k}, p \mid \mathbb{R}^{2} \geqslant 0 \Longrightarrow L(p) \geqslant 0$. Hilbert's theorem on sums of squares (cf. [15]) implies that $L: \mathscr{P}_{4} \longrightarrow \mathbb{R}$ is positive if and only if the moment matrix associated to $L$ is positive semidefinite. In this note, using $k=6$, we exhibit the first family of positive linear functionals $L: \mathscr{P}_{k} \rightarrow \mathbb{R}$ whose positivity cannot be derived from the positive semidefiniteness of the associated moment matrices, and which do not correspond to integration with respect to positive measures.

Mathematics subject classification (2010): 47A57, 44A60, 47A20.

Keywords and phrases: Truncated moment sequence, Riesz functional, $K$-positivity, moment matrix, representing measure.

\section{REFERENCES}

[1] C. Bayer And J. Teichmann, The proof of Tchakaloff's Theorem, Proc. Amer. Math. Soc., 134 (2006), 3035-3040.

[2] S. Berberian, Lectures in Functional Analysis and Operator Theory, Springer-Verlag, 1973.

[3] R. Curto And L. Fialkow, Recursiveness, positivity, and truncated moment problems, Houston J. Math. 17 (1991) 603-635.

[4] R. Curto And L. Fialkow, Solution of the truncated complex moment problem for flat data, Memoirs of the American Mathematical Society, 119 (1996), No. 568, Amer. Math. Soc. Providence, RI, 1996.

[5] R. Curto And L. Fialkow, Flat extensions of positive moment matrices: Relations in analytic or conjugate terms, Operator Th.: Adv. Appl. 104 (1998), 59-82.

[6] R. CURTO AND L. FiAlKow, Solution of the singular quartic moment problem, Journal of Operator Theory, 48 (2002), 315-354.

[7] R. CURTO AND L. FialKow, Truncated K-moment problems in several variables, Journal of Operator Theory, 54 (2005), 189-226.

[8] R. CuRto And L. Fialkow, Solution of the truncated hyperbolic moment problem, Integral Equations and Operator Theory, 52 (2005), 181-219.

[9] R. Curto And L. Fialkow, An analogue of the Riesz-Haviland Theorem for the truncated moment problem, J. Functional Analysis, 225 (2008), 2709-2731.

[10] L. FIalkow, Truncated multivariable moment problems with finite variety, J. Operator Theory, 60 (2008), 343-377.

[11] L. FiALKOW, Solution of the truncated moment problem with variety $y=x^{3}$, Trans. Amer. Math. Soc., 363 (2011), 3133-3165.

[12] L. Fialkow And J. NiE, Positivity of Riesz functionals and solutions of quadratic and quartic moment problems, J. Functional Analysis, 258, 1 (2010), 328-356.

[13] E. K. HaViland, On the momentum problem for distributions in more than one dimension II, Amer. J. Math., 58 (1936) 164-168.

[14] M. LAURENT, Sums of squares, moment matrices and optimization over polynomials, Emerging Applications of Algebraic Geometry, Vol. 149 of IMA Volumes in Mathematics and its Applications, M. Putinar and S. Sullivant (eds), Springer, pages 157-270, 2009. 
[15] B. ReZnick, Some concrete aspects of Hilbert's $17^{\text {th }}$ problem, in Contemp. Math., volume 253, pages 251-272, American Mathematical Society, 2000.

[16] B. REZNICK, On Hilbert's construction of positive polynomials, preprint, 2007.

[17] M. RIESZ, Sur le probleme des moments, troisieme note, Ark. Mat., 17 (1923), 1-52.

[18] K. SCHMÜDGEN, An example of a positive polynomial which is not a sum of squares of polynomials. A positive, but not strongly positive functional, Math. Nachr., 88 (1979), 385-390.

[19] K. SchmüDGEN, The K-moment problem for compact semi-algebraic sets, Math. Ann. 289 (1991), 203-206.

[20] V. TchaKaloff, Formules de cubatures mécanique à coefficients non négatifs, Bull. Sci. Math., (2)82 (1957), 123-134. 